\title{
The prognostic value of external vs internal pancreatic duct stents after pancreaticoduodenectomy in patients with $F R S \geq 4:$ a retrospective cohort study
}

\author{
Yuancong Jiang ${ }^{1 \dagger}$, Qin Chen ${ }^{2 \dagger}$, Yi Shao $^{3}$, Zhenzhen Gao ${ }^{1}$, Ming Jin ${ }^{1}$, Bingqiang Gao ${ }^{1}$, Bo Zhou ${ }^{1 *}$ \\ and Sheng Yan $^{1^{*}}$ (D)
}

\begin{abstract}
Background: The prognostic value of external vs internal pancreatic duct stents after pancreaticoduodenectomy remains controversial. This study aimed to evaluate the benefits of external and internal stents using the Fistula Risk Score system with regard to the incidence of clinically relevant postoperative pancreatic fistula.
\end{abstract}

Methods: A total of 382 patients who underwent pancreaticoduodenectomy with duct to mucosa pancreaticojejunostomy were retrospectively enrolled from January 2015 to October 2019. The receiver operating characteristic curve was performed for subgroup analysis of the patients at different levels of risk for pancreatic fistula.

Results: There were no significant differences in terms of pancreatic fistula or other postoperative complications. According to the receiver operating characteristic curve threshold of 3.5, 172 patients with a Fistula Risk Score $\geq 4$ and 210 patients with a Fistula Risk Score $<4$ were divided into separate groups. The number of valid cases was insufficient to support the subsequent research in patients with a Fistula Risk Score $<4$. In patients with a Fistula Risk Score $\geq 4$, the use of an external pancreatic duct stent was significantly more effective than the use of an internal stent, especially with regard to the risk for pancreatic fistula (Grade C) $(P=0.039)$, at ameliorating the incidence of clinically relevant postoperative pancreatic fistula $(P=0.019)$. Additionally, the incidence of lymphatic leakage was significantly higher in the external stent group compared with the internal stent group $(P=0.040)$.

Conclusions: Compared with internal stents, the use of an external stent could reduce the incidence of clinically relevant postoperative pancreatic fistula in patients with a Fistula Risk Score $\geq 4$. More large-scale prospective clinical trials are warranted to further clarify our results.

Keywords: Clinically relevant postoperative pancreatic fistula (CR-POPF), Pancreaticoduodenectomy, External pancreatic duct stent, Postoperative complications, Prognosis

*Correspondence: 1509004@zju.edu.cn; shengyan@zju.edu.cn †Yuancong Jiang and Qin Chen contributed equally to this work and shared the co-first author

${ }^{1}$ Division of Hepatobiliary and Pancreatic Surgery, Department of Surgery, The Second Affiliated Hospital, School of Medicine, Zhejiang University, 88 Jiefang Road, Hangzhou 310009, China

Full list of author information is available at the end of the article

\section{Background}

Pancreatic cancer, with its associated poor prognosis, is one of the most insidious and lethal cancers globally[1]. Indeed, pancreatic cancer has been listed as the fourth leading cause of cancer-related deaths in developed countries, and it may replace colorectal cancer as the second-leading cause of cancer-related deaths by $2030[2$, 
3]. Pancreaticoduodenectomy is the standard treatment for periampullary carcinoma, especially pancreatic head tumors [4]. However, the incidence of postoperative complications, especially postoperative pancreatic fistula, remains as high as $25 \%-50 \%$, which limits the dissemination of pancreaticoduodenectomy[5].

An external pancreatic duct stent is one of the methods used to prevent pancreatic fistula. A large number of studies, including prospective randomized trials as well as meta-analyses, have shown that external pancreatic duct stents significantly decrease the rate of pancreatic fistula and shorten the length of hospital stay[6-8]. Paradoxically, several studies have shown that external pancreatic duct stents have no effect and may even increase the incidence of postoperative pancreatic fistula[9-11]. In a meta-analysis, Dong et al. [12] observed that the use of an external pancreatic duct stent was associated with a significantly lower incidence of pancreatic fistula in patients at high risk for pancreatic fistula compared with an internal stent, but there was no definitive conclusion because of the low quality of the evidence[8].

In 2013, Callery et al. [13] proposed the Fistula Risk Score (FRS) according to the International Study Group of Pancreatic Surgery (ISGPS), which quantitatively validated the risk of pancreatic fistula and assessed the benefits of pancreatic duct stents in patients at different levels of risk. Moreover, ISGPS redefined the classification criteria for pancreatic fistula (Grade A) as a biochemical leak, which had no significant clinical impact on the clinical prognosis[14]. In addition, the position statement by ISGPS indicated that, due to the lack of high-quality evidence, the pancreatic duct stent was not routinely recommended during pancreaticoenteric anastomosis, but external stenting can be considered in high-risk glands[15]. Therefore, it is necessary to systematically re-investigate the safety and effectiveness of external pancreatic duct stents for the prevention and treatment of clinically relevant postoperative pancreatic fistula (CRPOPF) in both high-risk and low-risk patients. This retrospective study was conducted to evaluate the differences between external and internal pancreatic stents using the FRS scoring system, based on the hypothesis that the use of an external stent in high-risk patients could decrease the rates of CR-POPF compared with an internal stent after pancreaticoduodenectomy.

\section{Methods}

\section{Patients}

Clinical data of 382 patients who underwent pancreaticoduodenectomy in our pancreatic center from January 2015 to October 2019 were retrospectively collected. None of the patients had a past history of abdominal surgery or other cancers which might cause intra-abdominal adhesion. Patients who cannot perform pancreaticoduodenectomy due to severe comorbidities associated with the heart, lungs, liver, kidneys or organ metastases were excluded. Next, using the optimum cutoff point of the receiver operating characteristic (ROC) curve for subgroup analysis, these patients were separated into two groups: those at relatively high-risk for pancreatic fistula or those at relatively low-risk for pancreatic fistula.

\section{Surgical technique}

All pancreaticoduodenectomies were performed by the same surgical team at our pancreatic center throughout the study period. According to the preoperative laboratory results, medical imaging data and intraoperative conditions, the laparoscopic or open pancreaticoduodenectomy, or pylorus-preserving pancreaticoduodenectomy was performed at the discretion of the individual surgeon. Child's technique was implemented to achieve digestive tract reconstruction, and all patients underwent a duct-to-mucosa pancreaticojejunostomy. Furthermore, the internal pancreatic duct stent, a silicone catheter with multiple side pores, was inserted into the main pancreatic duct and the other end was placed in the jejunum cavity. Alternately, the external pancreatic duct stent left the other end exteriorized through the proximal jejunum via a small enterotomy that was fixed in the abdominal wall. All pancreaticoduodenectomies at our pancreatic center were implemented with external pancreatic duct stent or internal stent based on the experiences of the surgeons. Fibrin glue sealant was not applied during pancreaticojejunostomy anastomosis, and two abdominal drainage tubes were placed, one behind the biliary-enteric anastomosis and the other around the pancreatic-enteric anastomosis.

\section{Perioperative management}

Each patient underwent standardized perioperative management at the Second Affiliated Hospital, College of Medicine, Zhejiang University. Broad-spectrum antibiotics and anti-anaerobic drugs were used $72 \mathrm{~h}$ postoperatively to prevent infection after surgery. Jejunal-placed feeding tubes, nasogastric tubes and octreotide were routinely used. After bowel sounds were restored, the nasogastric tube was removed, and the normal diet was gradually resumed after 3-5 days.

Amylase levels in the serum and drainage fluid were routinely measured on postoperative days 3,5 and 7 . Then, a contrast-enhanced computed tomography was performed on postoperative days $5-7$ to screen for any possible complications. If there was no evidence of complications such as pancreatic fistula, bile leakage or infection, the abdominal drainage tube and external pancreatic duct stent were gradually removed during 
hospitalization. If a pancreatic fistula, a severe abdominal infection, or other severe complication was found, antibiotics were used based on blood cultures and drug sensitivity tests until the serum and drainage fluid amylase returned to normal levels and the external pancreatic duct stent was left in situ when the patient was discharged. One month after surgery, the external pancreatic duct stent was removed in the outpatient clinic.

\section{Study endpoints}

The primary endpoint of this retrospective study was the incidence of CR-POPF, which was graded according to the definition set forth by ISGPS in 2016[14]. The risk of CR-POPF was evaluated by FRS[13]. The secondary endpoints of this retrospective study included the incidence of delayed gastric emptying (DGE), postoperative pancreatic hemorrhage $(\mathrm{PPH})$, bile leakage, infection and mortality, which were graded according to the Clavien-Dindo Classification[16]. The incidence of complications was collected from medical records and a one-month outpatient follow-up period was implemented for patients with complications at the time of discharge.

\section{Statistical analysis}

SPSS 25.0 (International Business Machines Corporation, Armonk, New York) was used for all statistical analyses. The sample size was calculated using the Epi-Info companion. All continuous data were tested for normality using the Kolmogorov-Smirnov test. Normally distributed data were expressed as the mean \pm standard deviation and were analyzed using the unpaired, independent, two-tailed t-test. The other continuous data were expressed in interquartile ranges, and two samples rank sum tests were used. All categorical variables were tested using the chi-square test. Variables with $P$ values less than 0.05 in the univariate analysis were included in the multivariate analysis as independent risk factors. $P<0.05$ was considered statistically significant.

\section{Results}

Between January 2015 and October 2019, a total of 382 patients underwent pancreaticoduodenectomy and were enrolled in this study. Two hundred and ten patients had an FRS score of less than 4, 163 patients had an FRS score of 4-6, and 9 patients had an FRS score of greater than 7. Forty-five patients received an external pancreatic duct stent, and 337 patients received an internal pancreatic duct stent. There were no significant differences with regard to age $(P=0.202)$, gender $(P=0.734)$, body mass index (BMI) $(P=0.072)$, hypertension $(P=0.592)$, smoking status $(P=0.948)$, drinking $(P=0.057)$, weight loss $(P=0.292)$, intraoperative bleeding $(P=0.214)$ or pathological diagnosis $(P=0.428)$ between the two groups.
Compared to the internal stent group, the external stent group had a higher proportion of patients with a soft pancreas parenchyma $(P<0.001)$ and a small pancreatic duct diameter $(P<0.001)$, although there was a smaller proportion of patients with diabetes $(P=0.015)$. However, the incidence of pancreatic fistula or CR-POPF did not differ between the external and internal stent groups. The patient characteristics and postoperative complications are listed in Table 1 and the flow diagram for the study is shown in Additional file 1: Figure S1.

Considering that an external pancreatic duct stent is always used in patients at higher risk for pancreatic fistula, the ROC curve was used to find the threshold of the FRS. When FRS $=3.5$, the Youden's index is the highest, and the area under the curve (AUC) is 0.721 . The ROC curve is shown in Additional file 2: Figure S2. Therefore, a total of 172 patients with FRS $\geq 4$ were included in the subsequent research regarding the effect of an external stent in patients at relatively high-risk for pancreatic fistula. For the 210 patients with FRS $<4$, there were only 2 patients who received an external pancreatic duct stent. Concretely, a total of 19 patients with FRS $<4$ developed CR-POPF (8.6\%), with 0 patients in the external stent group (0.0\%) and 19 patients in the internal stent group (9.1\%). The number of valid cases was, therefore, insufficient to support the subsequent comparison of external stents and internal stents in patients at relatively low-risk for pancreatic fistula.

In the subgroup analysis of patients with FRS $\geq 4,43$ patients received an external pancreatic duct stent and 129 patients received an internal pancreatic duct stent. There were no significant differences with regard to the baseline characteristics between the two groups except for diabetes. Compared with patients in the internal stent group, the proportion of patients with diabetes in the external stent group was lower $(P=0.028)$. The characteristics of the patients at high-risk for a pancreatic fistula are listed in Table 2. The pancreaticoduodenectomy is confounded by many factors that affect the risk of pancreatic fistula, so we controlled for the anastomosis technique and the treatments that may be used to prevent pancreatic fistula, such as octreotide and fibrin glue sealant, to reduce subjective bias. As shown in Table 3, in contrast to the internal stent group, the external stent group had longer operation duration $(492.16 \pm 103.63$ vs $432.22 \pm 136.51, P=0.003)$, more combined vascular resections $(18.6 \%$ vs $3.9 \%, P=0.004)$, and a higher proportion of patients with pancreatic cancer or chronic pancreatitis $(P=0.002)$.

The overall complication rate was $27 / 43(62.7 \%)$ in the external stent group and 79/129 (61.2\%) in the internal stent group. Pancreatic fistula was the most common complication in our retrospective study. A total of 57 
Table 1 Baseline characteristics of external stent and internal stent group in all patients

\begin{tabular}{|c|c|c|c|}
\hline Characteristics & External stent group $\mathrm{N}=45$ & Internal stent group $\mathrm{N}=337$ & $P$-value \\
\hline Age (years) & $60(54-67)$ & $64(56-70)$ & $P=0.202$ \\
\hline Gender (M/F) & $27 / 18$ & $211 / 126$ & $P=0.734$ \\
\hline $\mathrm{BMI}\left(\mathrm{kg} / \mathrm{m}^{2}\right)$ & $23.01 \pm 3.14$ & $22.17 \pm 2.93$ & $P=0.072$ \\
\hline Hypertension & $12(26.7 \%)$ & $103(30.6 \%)$ & $P=0.592$ \\
\hline Diabetes & $1(2.2 \%)$ & $53(15.7 \%)$ & $P=0.015$ \\
\hline Smoke & $17(37.8 \%)$ & $129(38.3 \%)$ & $P=0.948$ \\
\hline Drink & $9(20.0 \%)$ & 115 (34.1\%) & $P=0.057$ \\
\hline Weight loss & $8(17.8 \%)$ & $84(24.9 \%)$ & $P=0.292$ \\
\hline Operative time (min) & $496.93 \pm 109.06$ & $425.39 \pm 142.77$ & $P=0.001$ \\
\hline Intraoperative bleeding $(\mathrm{mL})$ & $275(200-400)$ & $200(200-300)$ & $P=0.214$ \\
\hline Texture soft/Firm & $43 / 2$ & $179 / 158$ & $P<0.001$ \\
\hline Pancreatic duct diameter (cm) & $0.3(0.3-0.4)$ & $0.4(0.3-0.5)$ & $P<0.001$ \\
\hline Pathology & & & $P=0.428$ \\
\hline Pancreatic adenocarcinoma or pancreatitis & $15(33.3 \%)$ & $133(39.5 \%)$ & \\
\hline Ampullary, duodenal, cystic, islet cell, etc & $30(66.7 \%)$ & $204(60.5 \%)$ & \\
\hline Pancreatic fistula & $22(48.9 \%)$ & $128(38.0 \%)$ & $P=0.159$ \\
\hline CR-POPF & $8(17.8 \%)$ & $68(20.2 \%)$ & $P=0.705$ \\
\hline Grade A & $14(31.1 \%)$ & $60(17.8 \%)$ & \\
\hline Grade B & $8(17.8 \%)$ & $55(16.3 \%)$ & \\
\hline Grade C & $0(0 \%)$ & $13(3.9 \%)$ & $P=0.379$ \\
\hline
\end{tabular}

The italic values reflected that there was a significant difference between the two groups

Table 2 Baseline characteristics of external stent and internal stent group in high-risk of pancreatic fistula patients

\begin{tabular}{llll}
\hline Characteristics & $\begin{array}{l}\text { External stent } \\
\text { group N=43 }\end{array}$ & $\begin{array}{l}\text { Internal stent } \\
\text { group N=129 }\end{array}$ & $P$-value \\
\hline Age (years) & $60(56-67)$ & $62(56-70)$ & $P=0.406$ \\
Gender (M/F) & $27 / 16$ & $84 / 45$ & $P=0.783$ \\
BMI (kg/m²) & $23.03 \pm 3.18$ & $22.53 \pm 2.79$ & $P=0.334$ \\
Hypertension & $11(25.6 \%)$ & $39(30.2 \%)$ & $P=0.561$ \\
Diabetes & $1(2.3 \%)$ & $19(14.7 \%)$ & $P=0.028$ \\
Smoke & $16(37.2 \%)$ & $47(36.4 \%)$ & $P=0.927$ \\
Drink & $8(18.6 \%)$ & $37(28.7 \%)$ & $P=0.193$ \\
Weight Loss & $8(18.6 \%)$ & $23(17.8 \%)$ & $P=0.909$ \\
\hline
\end{tabular}

The italic values reflected that there was a significant difference between the two groups

patients developed CR-POPF (33.1\%), with 8 patients in the external stent group and 49 patients in the internal stent group. External pancreatic duct stents were significantly more effective than internal stents at ameliorating CR-POPF (38.0\% vs $18.6 \%, P=0.019)$. Notably, the incidence of pancreatic fistula (Grade C) in the external stent group was significantly lower than in the internal stent group $(0.0 \%$ vs $9.3 \%, P=0.039)$. Additionally, 46 patients experienced an abdominal infection (26.7\%), and while the incidence of abdominal infection in the external stent group was lower than in the internal stent group $(20.9 \%$ vs $28.7 \%, P=0.320$ ), the difference was not statistically significant. Twenty-eight patients experienced lymphatic leakage (16.3\%), including 11 patients in the external stent group and 16 patients in the internal stent group. Lymphatic leakage in the external stent group tended to be significantly greater than in the internal stent group (25.6\% vs $12.4 \%, P=0.040$ ).

The incidence of other complications was low. A total of 27 patients experienced PPH (15.7\%), 21 patients required additional surgery (12.2\%), 16 patients contracted pulmonary infections (9.3\%), 14 patients were found to have DGE (8.1\%), 11 patients developed pancreatic pseudocysts $(6.4 \%), 9$ patients experienced bile leakage (5.2\%), 9 patients died during hospitalization (5.2\%), 7 patients developed intestinal obstruction (4.1\%) and stent-related complications occurred in 3 patients (1.7\%). No statistically significant difference in complications was found between the two groups. The postoperative complications between the external and internal stent groups are listed in Table 4.

Univariate analysis revealed that BMI $(P=0.009)$, hypertension $(P=0.009)$, pancreatic duct stent $(P=0.022)$, blood transfusion $(P=0.020)$ and pancreatic duct diameter $(P=0.014)$ were identified as significant risk factors for CR-POPF. Next, multivariate analysis was used to explore the independent risk factors 
Table 3 Comparison of intraoperative variables between external stent and internal stent group in high-risk of pancreatic fistula patients

\begin{tabular}{|c|c|c|c|}
\hline Variables & External stent group $\mathrm{N}=43$ & Internal stent group $\mathrm{N}=129$ & $P$-value \\
\hline Preoperative biliary drainage & $12(27.9 \%)$ & $27(20.9 \%)$ & $P=0.344$ \\
\hline Percutaneous/endoscopic & $7 / 5$ & $7 / 20$ & \\
\hline Preoperative total bilirubin $(\mu \mathrm{mol} / \mathrm{L})$ & $17.5(11-135)$ & $28(13.5-121.5)$ & $P=0.581$ \\
\hline Preoperative direct bilirubin $(\mu \mathrm{mol} / \mathrm{L})$ & $9.9(4-107.3)$ & $9.7(3-79.7)$ & $P=0.535$ \\
\hline Preoperative CA $199(\mathrm{U} / \mathrm{mL})$ & $20.7(5.8-191)$ & $27(7.9-156.2)$ & $P=0.965$ \\
\hline Operative time (min) & $492.16 \pm 103.63$ & $432.22 \pm 136.51$ & $P=0.003$ \\
\hline Intraoperative bleeding (mL) & $225(200-400)$ & $200(200-400)$ & $P=0.712$ \\
\hline$\leq 400$ & 33 & 101 & \\
\hline$>400$ & 10 & 28 & \\
\hline Vascular resection & $8(18.6 \%)$ & $5(3.9 \%)$ & $P=0.004$ \\
\hline Blood transfusion & $17(39.5 \%)$ & $38(29.5 \%)$ & $P=0.220$ \\
\hline Texture soft/Firm & $43 / 0$ & $121 / 8$ & $P=0.203$ \\
\hline Pancreatic duct diameter (cm) & $0.3(0.3-0.4)$ & $0.3(0.3-0.3)$ & $P=0.205$ \\
\hline 0.1 & 1 & 0 & \\
\hline 0.2 & 6 & 26 & \\
\hline 0.3 & 22 & 76 & \\
\hline 0.4 & 12 & 22 & \\
\hline 0.5 & 2 & 4 & \\
\hline$>0.5$ & 0 & 1 & \\
\hline Pathology & & & $P=0.002$ \\
\hline Pancreatic adenocarcinoma or pancreatitis & 13 & 14 & \\
\hline Ampullary, duodenal, cystic, islet cell, etc & 30 & 115 & \\
\hline Pancreatic adenocarcinoma & 12 & 12 & \\
\hline Cholangiocarcinoma & 2 & 16 & \\
\hline Duodenal carcinoma & 16 & 39 & \\
\hline Chronic pancreatitis & 1 & 2 & \\
\hline Pancreatic cystic tumor & 1 & 19 & \\
\hline Pancreatic neuroendocrine tumor & 1 & 3 & \\
\hline Ampullary carcinoma & 5 & 26 & \\
\hline Others & 5 & 12 & \\
\hline
\end{tabular}

The italic values reflected that there was a significant difference between the two groups

for CR-POPF after pancreaticoduodenectomy. As shown in Table 5, high BMI (OR 1.175, $P=0.013$ ), the use of an internal stent (OR 3.376, $P=0.011$ ), receipt of a blood transfusion (OR 2.777, $P=0.008)$ and a narrow pancreatic duct (OR 0.003, $P=0.024$ ) were independent risk factors predictive of CR-POPF.

\section{Discussion}

Pancreatic fistula is one of the most common and serious complications after pancreaticoduodenectomy and may lead to potentially fatal complications. In 1999, Roder et al. [7] reported that the use of external pancreatic duct stents could reduce the incidence of pancreatic fistula from $29.3 \%$ to $6.8 \%$ and shorten the length of hospital stay, which demonstrated the capacity of external pancreatic duct stents to be an effective method for pancreatic fistula prevention. The reasons for the current use of external stents for the treatment of pancreatic fistula are as follows: First, the patency of the pancreatic duct is supported by external pancreatic stents so that the pancreatic enzymes that accumulate in the jejunum after surgery can be expelled expediently, avoiding activation by enterokinase, and therefore preventing further digestion of the residual pancreas. Second, the successful discharge of pancreatic juices relieves the tension on the pancreatic-enteric anastomosis and promotes postoperative anastomotic healing. Moreover, the use of external pancreatic duct stents allows for a more precise placement of the sutures during pancreaticoduodenectomy to avoid suture injury and further iatrogenic damage[6, $7,17]$. Compared with simply stenting the pancreatic 
Table 4 Comparison of postoperative complications between external stent and internal stent group in highrisk of pancreatic fistula patients

\begin{tabular}{lcll}
\hline $\begin{array}{l}\text { Postoperative } \\
\text { complications }\end{array}$ & $\begin{array}{c}\text { External stent } \\
\text { group N=43 }\end{array}$ & $\begin{array}{l}\text { Internal stent } \\
\text { group N=129 }\end{array}$ & $P$-value \\
\hline Pancreatic fistula & $22(51.2 \%)$ & $78(60.5 \%)$ & $P=0.284$ \\
CR-POPF & $8(18.6 \%)$ & $49(38.0 \%)$ & $P=0.019$ \\
Grade A & $14(32.6 \%)$ & $29(22.5 \%)$ & $P=0.186$ \\
Grade B & $8(18.6 \%)$ & $37(28.7 \%)$ & $P=0.193$ \\
Grade C & $0(0.0 \%)$ & $12(9.3 \%)$ & $P=0.039$ \\
Delayed gastric emptying & $3(7.0 \%)$ & $11(8.5 \%)$ & $P=1.000$ \\
Intra-abdominal bleeding & $6(14.0 \%)$ & $21(16.3 \%)$ & $P=0.717$ \\
Lymphatic leakage & $11(25.6 \%)$ & $16(12.4 \%)$ & $P=0.040$ \\
Bile leakage & $4(9.3 \%)$ & $5(3.9 \%)$ & $P=0.230$ \\
Intestinal obstruction & $2(4.7 \%)$ & $5(3.9 \%)$ & $P=1.000$ \\
Pancreatic pseudocyst & $3(7.0 \%)$ & $8(6.2 \%)$ & $P=1.000$ \\
Pulmonary infection & $3(7.0 \%)$ & $13(10.1 \%)$ & $P=0.764$ \\
Abdominal infection & $9(20.9 \%)$ & $37(28.7 \%)$ & $P=0.320$ \\
Re-operation & $5(11.6 \%)$ & $16(12.4 \%)$ & $P=0.893$ \\
In-hospital mortality & $1(2.3 \%)$ & $8(6.2 \%)$ & $P=0.453$ \\
Stent-related complications & $2(4.7 \%)$ & $1(0.8 \%)$ & $P=0.155$ \\
Postoperative hospital stay (day) & $18(14-25)$ & $17(13-26)$ & $P=0.715$ \\
\hline
\end{tabular}

The italic values reflected that there was a significant difference between the two groups duct into the jejunum cavity, the external stent minimizes the digestive erosion of the residual pancreas and fully eliminates pancreatic juices from the anastomosis. In addition, the volume of pancreatic juice drainage can be routinely measured as a precaution against premature detachment of the pancreatic duct stent following pancreaticojejunostomy. Whereas, DGE may result from the loss of a large amount of fluid and digestive enzymes, and better nutritional support and volume maintenance is therefore required. Additionally, compared with internal stents, external stents are more likely to cause stentrelated complications, such as bending, displacement, shedding, or blockage of the drainage tube, as well as peritonitis, chronic pancreatitis or stenosis after removal of the stent $[18,19]$. Thus, the benefit of an external over an internal stent for the prevention of CR-POPF remains controversial[10, 20, 21].

Based on the new definition of pancreatic fistula and the lack of explicit selectivity with validated FRS for high-risk patients receiving an external pancreatic duct stent in the current literature, some patients at low risk of pancreatic fistula may not benefit from an external stent, resulting in insufficient results. According to the ROC curve, the FRS threshold was found to be 3.5. Thus, in order to compare

Table 5 Univariable and multivariable analysis of risk factors for clinically relevant postoperative pancreatic fistula in high-risk of pancreatic fistula patients

\begin{tabular}{|c|c|c|c|c|c|c|}
\hline & \multicolumn{3}{|c|}{ Univariable analysis } & \multicolumn{3}{|c|}{ Multivariable analysis } \\
\hline & OR & $95 \% \mathrm{Cl}$ & $P$-value & OR & $95 \% \mathrm{Cl}$ & $P$-value \\
\hline Age (years) & 1.000 & $0.969-1.032$ & 0.999 & & & \\
\hline Female (ref: male) & 0.976 & $0.502-1.896$ & 0.942 & & & \\
\hline BMI $\left(\mathrm{kg} / \mathrm{m}^{2}\right)$ & 1.167 & $1.040-1.311$ & 0.009 & 1.175 & $1.034-1.335$ & 0.013 \\
\hline Hypertension & 2.490 & $1.257-4.932$ & 0.009 & 1.791 & $0.838-3.829$ & 0.133 \\
\hline Diabetes & 2.234 & $0.871-5.728$ & 0.094 & & & \\
\hline Smoke & 0.718 & $0.366-1.406$ & 0.334 & & & \\
\hline Drink & 0.663 & $0.312-1.409$ & 0.285 & & & \\
\hline Weight Loss & 1.262 & $0.847-1.880$ & 0.253 & & & \\
\hline Preoperative biliary drainage & 1.354 & $0.645-2.843$ & 0.423 & & & \\
\hline Preoperative total bilirubin ( $\mu \mathrm{mol} / \mathrm{L})(\mathrm{ref}: \leq 21)$ & 1.415 & $0.738-2.715$ & 0.296 & & & \\
\hline Preoperative direct bilirubin $(\mu \mathrm{mol} / \mathrm{L})(\mathrm{ref}: \leq 8)$ & 1.613 & $0.848-3.069$ & 0.145 & & & \\
\hline Preoperative CA $199(\mathrm{U} / \mathrm{mL})($ ref: $\leq 37)$ & 0.791 & $0.416-1.506$ & 0.476 & & & \\
\hline Operative time (min) & 0.999 & $0.997-1.002$ & 0.463 & & & \\
\hline Intraoperative bleeding (mL) & 1.000 & $0.999-1.001$ & 0.815 & & & \\
\hline Vascular resection & 0.583 & $0.154-2.208$ & 0.427 & & & \\
\hline Internal stent (ref: external stent) & 2.680 & $1.149-6.247$ & 0.022 & 3.376 & $1.318-8.646$ & 0.011 \\
\hline Blood transfusion & 2.214 & $1.134-4.319$ & 0.020 & 2.777 & $1.310-5.887$ & 0.008 \\
\hline Texture Firm (ref: soft) & 0.276 & $0.033-2.295$ & 0.233 & & & \\
\hline Pancreatic duct diameter $(\mathrm{cm})$ & 0.003 & $0.000-0.319$ & 0.014 & 0.003 & $0.000-0.456$ & 0.024 \\
\hline $\begin{array}{l}\text { Pancreatic adenocarcinoma or pancreatitis (ref: amp- } \\
\text { ullary, duodenal, cystic, islet cell, etc.) }\end{array}$ & 1.480 & $0.636-3.440$ & 0.363 & & & \\
\hline
\end{tabular}

The italic values reflected that there was a significant difference between the two groups 
the efficacy of external pancreatic duct stents with internal stents in the prevention and treatment of CR-POPF, we combined the FRS system to quantitatively evaluate the effect of the two types of pancreatic stents on patients at different levels of risk for pancreatic fistula. Eventually, 172 patients with $F R S \geq 4$ were enrolled to evaluate the differences between external and internal stents.

Our retrospective study provided two important findings. First, for patients at high-risk of pancreatic fistula with $\mathrm{FRS} \geq 4$, the use of an external stent was associated with a lower rate of CR-POPF, which is consistent with the statement by the ISGPS. Moreover, external stents exerted a more pronounced effect than the internal stents on the prevention of pancreatic fistula (Grade C). No pancreatic fistula (Grade C) and only 8 pancreatic fistulas (Grade B) occurred in the 43 patients who received an external stent. Interestingly, there was no significant difference in the overall incidence of pancreatic fistula between the two groups, and the proportion of patients with pancreatic fistula (Grade A) was even higher in the external stent group than in the internal stent group, which may explain the uncertain results in the previous studies. Second, compared with the patients who received an internal stent, the incidence of lymphatic leakage in patients with external pancreatic duct stents was significantly increased. Due to the higher proportion of patients with pancreatic cancer in the external stent group, more vascular resections and more extensive lymph node dissections were required to achieve curative (R0) resection, which meant that patients who received external stenting required longer hospital stays for the conservative treatment of lymphatic leakage and subsequent complications, such as electrolyte disturbances or abdominal infections. Collectively, the association between pancreatic stent and lymphatic leakage was not clear, but it appeared related to the pathology type and extension of the operation rather than the presence of an external stent.

Although our retrospective study indicated that the use of an external pancreatic duct stent has an excellent preventive effect against CR-POPF, especially pancreatic fistula (Grade C) in patients with FRS $\geq 4$, there remain some limitations in our research. First, due to the retrospective design without randomized allocation, there may have been some bias in the selection of the controls and baseline characteristics between external stent and internal stent group in all patients or in high-risk pancreatic fistula patients. This bias may be caused by the discretion of the individual surgeon which may have an impact on the outcome. In clinical practice, the external stent is more likely to be used in patients with soft pancreatic parenchyma and small pancreatic ducts. As for the bias of diabetes, a subgroup analysis was performed to resolve the selection bias. As shown in the Additional file 3: Table S1 and Additional file 4: Table S2, consistent results were observed, with favorable outcomes of external stents in patients without diabetes. However, the cases were not valid to support the subsequent comparison between external stents vs internal stents in patients with diabetes. Besides, to reduce the selection bias and enhance the accuracy of our research, we conducted a rigorous control of the technique of anastomosis and application of octreotide or other measures, and standardized perioperative management was performed according to our hospital's policies. However, the outcomes following placement of the two stents were still impacted by the surgeons' clinical experience and subjective choices. Second, as the external stent was more frequently used in patients with high FRS scores, the sample size was insufficient to detect a statistically significant effect in patients with FRS $<4$. Third, although the FRS system is currently a classic and broadly deployed risk prediction tool for CR-POPF worldwide, it still has its shortcomings. There are many up-to-date scoring systems available, such as the alternative Fistula Risk Score or the Updated Alternative Fistula Risk Score, to validate and further expand the FRS's scope of application[22]. More large multicenter randomized prospective clinical trials are warranted based on the latest pancreatic fistula risk scoring system to verify the effectiveness of the implementation of external pancreatic duct stents in the prevention and treatment of CR-POPF in patients at different levels of risk for pancreatic fistula.

\section{Conclusions}

The use of external pancreatic duct stents could reduce the incidence of CR-POPF in patients with FRS $\geq 4$, but the incidence of lymphatic leakage may be increased. The relationship between external stent placement and lymphatic leakage still needed further investigation. However, it is still recommended that patients with FRS $\geq 4$ receive an external stent to avoid the possibly life-threatening occurrence of CR-POPF.

\section{Supplementary Information}

The online version contains supplementary material available at https://doi. org/10.1186/s12893-021-01074-w.

Additional file 1: Figure S1. Flow diagram for the study. FRS, Fistula Risk Score.

Additional file 2: Figure S2. The ROC curve predicts the FRS score threshold. The area under the ROC curve (AUC) is 0.721 . When FRS is 3.5, the sensitivity and specificity are 0.75 and 0.376 , respectively. ROC, the receiver operating characteristic curve; FRS, Fistula Risk Score.

Additional file 3: Table S1. Baseline characteristics of External stent and Internal stent group in all patients without diabetes.

Additional file 4: Table S2. Baseline characteristics of External stent and Internal stent group in high-risk of pancreatic fistula patients without diabetes. 


\section{Abbreviations}

FRS: Fistula Risk Score; ISGPS: International Study Group of Pancreatic Surgery; CR-POPF: Clinically relevant postoperative pancreatic fistula; ROC: Receiver operating characteristic; DGE: Delayed gastric emptying; PPH: Postoperative pancreatic hemorrhage; BMl: Body mass index; AUC: Area under the curve.

\section{Acknowledgements}

Not applicable.

\section{Authors' contributions}

All authors helped to perform the research and met the ICMJE recommendations. YCJ and QC: manuscript writing, performing data collect, data analysis, revising the manuscript and final approval of manuscript; YS and ZZG: manuscript writing, performing data collect, data analysis, organizing the data and figures and final approval of manuscript; MJ and BQG: manuscript writing, performing data collect, data analysis and final approval of manuscript; BZ and SY: performing the surgery, writing the manuscript, drafting conception and design, final approval of manuscript. All authors have read and approved the manuscript.

\section{Funding}

This work was supported by the National Natural Science Foundation of China under Grant number [81572975]; and the Key research and development project of science and technology department of Zhejiang under Grant number [2015C03053]. The funders had no role in the design of the study and collection, analysis, and interpretation of data and in writing the manuscript.

\section{Availability of data and materials}

The data used to support the findings of this study are available from the corresponding author upon reasonable request.

\section{Ethics approval and consent to participate}

This study was reviewed and approved by the Research Ethics Committee of the Second Affiliated Hospital, College of Medicine, Zhejiang University (approval number 2020-327). All patients enrolled completed the informed consent form.

\section{Consent for publication}

Not applicable.

\section{Competing interests}

The authors declarethat they have no competing interests.

\section{Author details}

1 Division of Hepatobiliary and Pancreatic Surgery, Department of Surgery, The Second Affiliated Hospital, School of Medicine, Zhejiang University, 88 Jiefang Road, Hangzhou 310009, China. ${ }^{2}$ Cancer Institute (Key Laboratory of Cancer Prevention and Intervention, China National Ministry of Education, Key Laboratory of Molecular Biology in Medical Sciences, Zhejiang Province, China), The Second Affiliated Hospital, School of Medicine, Zhejiang University, Hangzhou, China. ${ }^{3}$ Division of Hepatobiliary and Pancreatic Surgery, Department of Surgery, The First Affiliated Hospital, College of Medicine, Zhejiang University, Hangzhou, China.

Received: 25 August 2020 Accepted: 25 January 2021

Published online: 12 February 2021

\section{Reference:s}

1. Bray F, Ferlay J, Soerjomataram I, Siegel RL, Torre LA, Jemal A. Global cancer statistics 2018: GLOBOCAN estimates of incidence and mortality worldwide for 36 cancers in 185 countries. CA Cancer J Clin. 2018;68(6):394-424.

2. Strobel O, Neoptolemos J, Jager D, Buchler MW. Optimizing the outcomes of pancreatic cancer surgery. Nat Rev Clin Oncol. 2019;16(1):11-26.

3. Rahib L, Smith BD, Aizenberg R, Rosenzweig AB, Fleshman JM, Matrisian LM. Projecting cancer incidence and deaths to 2030: the unexpected burden of thyroid, liver, and pancreas cancers in the United States. Can Res. 2014;74(11):2913-21.

4. Donahue TR, Reber HA. Surgical management of pancreatic cancer-pancreaticoduodenectomy. Semin Oncol. 2015;42(1):98-109.
5. Guerrini GP, Soliani P, D'Amico G, Di Benedetto F, Negri M, Piccoli M, Ruffo G, Orti-Rodriguez RJ, Pissanou T, Fusai G. Pancreaticojejunostomy versus pancreaticogastrostomy after pancreaticoduodenectomy: an up-to-date meta-analysis. J Invest Surg . 2016;29(3):175-84.

6. Poon RT, Fan ST, Lo CM, Ng KK, Yuen WK, Yeung C, Wong J. External drainage of pancreatic duct with a stent to reduce leakage rate of pancreaticojejunostomy after pancreaticoduodenectomy: a prospective randomized trial. Ann Surg. 2007;246(3):425-33.

7. Roder JD, Stein HJ, Bottcher KA, Busch R, Heidecke CD, Siewert JR. Stented versus nonstented pancreaticojejunostomy after pancreatoduodenectomy: a prospective study. Ann Surg. 1999;229(1):41-8.

8. Jiang Y, Chen Q, Wang Z, Shao Y, Hu C, Ding Y, Shen Z, Jin M, Yan S. The prognostic value of external vs internal pancreatic duct stents in CR-POPF after pancreaticoduodenectomy: a systematic review and meta-analysis. J Invest Surg. 2020. https://doi.org/10.1080/08941939.2019.1691687.

9. Suzuki S, Kaji S, Koike N, Harada N, Tanaka S, Hayashi T, Suzuki M, Hanyu F. Pancreaticojejunostomy of duct to mucosa anastomosis can be performed more safely without than with a stenting tube. Am J Surg. 2009;198(1):51-4.

10. Xiong JJ, Altaf K, Mukherjee R, Huang W, Hu WM, Li A, Ke NW, Liu XB. Systematic review and meta-analysis of outcomes after intraoperative pancreatic duct stent placement during pancreaticoduodenectomy. Br J Surg. 2012;99(8):1050-61.

11. Kaman L, Nusrath S, Dahiya D, Duseja A, Vyas S, Saini V. External stenting of pancreaticojejunostomy anastomosis and pancreatic duct after pancreaticoduodenectomy. Updates Surg. 2012;64(4):257-64.

12. Dong Z, Xu J, Wang Z, Petrov MS. Stents for the prevention of pancreatic fistula following pancreaticoduodenectomy. Cochrane Database Syst Rev. 2013. https://doi.org/10.1002/14651858.CD008914.pub2.

13. Callery MP, Pratt WB, Kent TS, Chaikof EL, Vollmer CM Jr. A prospectively validated clinical risk score accurately predicts pancreatic fistula after pancreatoduodenectomy. J Am Coll Surg. 2013;216(1):1-14.

14. Bassi C, Marchegiani G, Dervenis C, Sarr M, Abu Hilal M, Adham M, Allen P, Andersson R, Asbun HJ, Besselink MG, et al. The 2016 update of the International Study Group (ISGPS) definition and grading of postoperative pancreatic fistula: 11 Years After. Surgery. 2017;161(3):584-91.

15. Shrikhande $S V$, Sivasanker $M$, Vollmer $C M$, Friess $H$, Besselink MG, Fingerhut $A$, Yeo CJ, Fernandez-delCastillo C, Dervenis C, Halloran C, et al. Pancreatic anastomosis after pancreatoduodenectomy: A position statement by the International Study Group of Pancreatic Surgery (ISGPS). Surgery. 2017;161(5):1221-34.

16. Dindo D, Demartines N, Clavien PA. Classification of surgical complications: a new proposal with evaluation in a cohort of 6336 patients and results of a survey. Ann Surg. 2004;240(2):205-13.

17. Sriussadaporn S, Pak-Art R, Sriussadaporn S, Kritayakirana K, Prichayudh S. Pancreaticoduodenectomy with external drainage of the pancreatic remnant. Asian J Surg. 2008;31(4):167-73.

18. Winter JM, Cameron JL, Campbell KA, Chang DC, Riall TS, Schulick RD, Choti MA, Coleman J, Hodgin MB, Sauter PK, et al. Does pancreatic duct stenting decrease the rate of pancreatic fistula following pancreaticoduodenectomy? Results of a prospective randomized trial. J Gastrointest Surg. 2006;10(9):1280-90.

19. Ohwada S, Tanahashi Y, Ogawa T, Kawate S, Hamada K, Tago Kl, Yamada T, Morishita Y. In situ vs ex situ pancreatic duct stents of duct-to-mucosa pancreaticojejunostomy after pancreaticoduodenectomy with billroth I-type reconstruction. Arch Surg. 2002;137(11):1289-93.

20. Hong S, Wang H, Yang S, Yang K. External stent versus no stent for pancreaticojejunostomy: a meta-analysis of randomized controlled trials. J Gastrointest Surg. 2013;17(8):1516-25.

21. Ke FY, Wu XS, Zhang Y, Zhang HC, Weng MZ, Liu YB, Wolfgang C, Gong W. Comparison of postoperative complications between internal and external pancreatic duct stenting during pancreaticoduodenectomy: a meta-analysis. Chin J Cancer Res Chung-kuo yen cheng yen chiu. 2015;27(4):397-407.

22. Mungroop TH, Klompmaker S, Wellner UF, Steyerberg EW, Coratti A, D'Hondt M, de Pastena M, Dokmak S, Khatov I, Saint-Marc O, et al. Updated alternative fistula risk score (ua-FRS) to include minimally invasive pancreatoduodenectomy: pan-european validation. Ann Surg. 2019. https://doi. org/10.1097/SLA.0000000000003234.

\section{Publisher's Note}

Springer Nature remains neutral with regard to jurisdictional claims in published maps and institutional affiliations. 